Asian J. Med. Biol. Res. 2020, 6 (2), 222-228; doi: 10.3329/ajmbr.v6i2.48053

\author{
Asian Journal of \\ Medical and Biological Research \\ ISSN 2411-4472 (Print) 2412-5571 (Online) \\ www.ebupress.com/journal/ajmbr
}

\title{
Article \\ Isolation and identification of Rhizobium from non-saline coastal soils of Bangladesh and preparation of mother culture
}

Md. Rafiq Uddin ${ }^{1 *}$, Mosa. Farjana Tasmin ${ }^{1}$, Md. Shahin Hossin ${ }^{1}$, Md. Mainul Islam Rashad ${ }^{2}$, Md. Fazlul Hoque $^{1}$ and Md. Kabirul Islam ${ }^{1}$

${ }^{1}$ Department of Soil Science, Patuakhali Science and Technology University, Dumki, Patuakhali-8602, Bangladesh

${ }^{2}$ Department of Genetics and Plant Breeding, Patuakhali Science and Technology University, Dumki, Patuakhali-8602, Bangladesh

"Corresponding author: Md. Rafiq Uddin, Department of Soil Science, Faculty of Agriculture, Patuakhali Science and Technology University, Dumki, Patuakhali-8602, Bangladesh. E-mail: rafiq_ssc@pstu.ac.bd

Received: 30 April 2020/Accepted: 30 May 2020/ Published: 30 June 2020

\begin{abstract}
Nitrogen is the essential mineral macro nutrients which are required for the maximum magnification and yield of agriculturally paramount crops. Microbial inoculants may supplement and abbreviate the dependency on synthetic costly $\mathrm{N}$-fertilizers in reverence of crop yield. The categorical objectives of the research works are to isolate and identify the Rhizobium from culled soils. The ability of soil microorganisms to fix atmospheric nitrogen is an important trait in promoting plant growth and increasing crop yield. The study was conducted for the isolation and identification of nitrogen fixing bacteria from saline and non-saline soils of different locations of tidal floodplain region of Bangladesh. Six Rhizobium strains were isolated and purified. The isolates were preliminary identified on the basis of their morphological and biochemical characteristics. Based on the results, it can be concluded that the isolates possess great potential to be developed as biofertilizers to enhance soil fertility and plant growth. However, their performance under green house and field conditions should be assessed afore being recommended for biofertilizer production and their applications.
\end{abstract}

Keywords: biofertilizer; non-saline soils; Rhizobium

\section{Introduction}

Nitrogen is an essential nutrient for plant growth and development. Plants usually depend upon combined, or fixed, forms of nitrogen, such as ammonia and nitrate because it is unavailable in its most prevalent form as atmospheric nitrogen. Much of this nitrogen is provided to cropping systems in the form of industrially produced nitrogen fertilizers. Use of these fertilizers has led to worldwide ecological problems as well as affects the human health (Vitousek, 1997). Biological nitrogen fixation (BNF) is the cheapest and environment friendly procedure in which nitrogen fixing micro-organisms, interacting with leguminous plants, fix aerobic nitrogen into soil (Franche et al., 2009).

Rhizobium is the most well-known species of a group of bacteria that acts as the primary symbiotic fixer of nitrogen. These bacteria can infect the roots of leguminous plants, leading to the formation of lumps or nodules where the nitrogen fixation takes place. The bacterium's enzyme system supplies a constant source of reduced nitrogen to the host plant and the plant furnishes nutrients and energy for the activities of the bacterium. Rhizibium bacteria stimulate the growth of leguminous plants and they are able to fix atmospheric nitrogen into soil by interacting symbiotically with leguminous plants, using the nitrogenase enzyme complex (Kiers et al., 2003).

Rhizobium is the soil microorganisms that can survive in the soil or forms a symbiotic association with the host legume. The most convenient method of obtaining Rhizobium from nature is by isolation from root nodules. In contrary to popular belief, many of the bacteriods in nodule are viable. It is impractical to isolate rhizobia 
directly from the soil because of their fastidious growth. The primary objective of the proposed research is to develop a cheap organic nitrogen fertilizer that could supplement synthetic nitrogen fertilizer.

\section{Materials and Methods}

\subsection{Sampling site}

Selected sampling sites were Charfashion upazilla of Bhola district under AEZ 18 (Young Meghna Estuarine Floodplain) and Dumki upazilla of Patuakhali district under AEZ 13 (Ganges Tidal Floodplain) of Bangladesh.

\subsection{Collection of soil samples}

For isolation of rhizobia, soil samples were collected from selected areas. Ten surface soil samples were collected from each location. For the convenience of discussion, the 6 soil samples are referred to as soil -1 , soil-2, soil-3, soil-4, soil-5 and soil-6 and these soil samples were collected from Aslampur, Ginnagor, Osmangonj of Charfashion upazila in Bhola district and Srirampur, PSTU Farm, Jamla of Dumki upazila in Patuakhali, respectively.

\subsection{Preparation of the soil sample}

Some portions of collected soil sample were kept in refrigerator at $4^{0} \mathrm{C}$ for isolation of bacteria. The rest portions of soil samples were then air dried ground to pass through a $2 \mathrm{~mm}$ sieve and then mixed to from a composite sample. Then these composite samples were kept in clean and sterilized bottles for physical and chemical analysis.

\subsection{Experimental site}

The laboratory experiment was conducted at the Department of Soil Science and Central Laboratory, Patuakhali Science and Technology University, Dumki, Patuakhali during July 2014 to June 2015.

\subsection{Soil analysis}

The initial soil samples were analyzed for physical and chemical characteristics. The physical characteristics includes textural class and the chemical properties include soil $\mathrm{pH}$, electrical conductivity, organic matter, total $\mathrm{N}$, Exchangeable K, available P and S content. Results of this analysis have been presented in Table 1.

Table 1. Physical and chemical characteristics of collected soils.

\begin{tabular}{lllllll}
\hline Properties of soils & Soil-1 & Soil-2 & Soil-3 & Soil-4 & Soil-5 & Soil-6 \\
\hline \%Sand & 19.2 & 19.5 & 19.3 & 21.7 & 20.9 & 21.5 \\
\%Silt & 67 & 66 & 68 & 70.75 & 70.86 & 70.92 \\
\%Clay & 13.8 & 13.9 & 13.6 & 7.55 & 7.75 & 7.68 \\
pH $\left(\mathrm{H}_{2} \mathrm{O}\right)$ & 7.65 & 7.88 & 7.75 & 6.56 & 5.98 & 6.43 \\
EC & 0.67 & 0.80 & 0.73 & 0.12 & 0.20 & 0.17 \\
\%C & 1.070 & 1.069 & 1.081 & 1.091 & 1.079 & 1.086 \\
$\%$ OM & 1.845 & 1.762 & 1.812 & 1.880 & 1.743 & 1.810 \\
\% & 0.047 & 0.042 & 0.062 & 0.014 & 0.020 & 0.019 \\
P(ppm) & 8.73 & 8.67 & 8.59 & 10.65 & 10.76 & 10.63 \\
S(ppm) & 32.77 & 32.69 & 32.82 & 13.52 & 13.35 & 12.98 \\
K(meq/100g) & 0.989 & 0.974 & 0.871 & 1.209 & 1.214 & 1.192 \\
\hline
\end{tabular}

\subsection{Culture media}

Yeast mannitol agar media were used for culture of Rhizobium.

\subsection{Method of isolation}

Enrichment culture technique (in liquid medium) was used for isolation of bacteria.

\subsection{Composition of yeast extract mannitol agar}

Mannitol - $10.0 \mathrm{~g}, \mathrm{~K}_{2} \mathrm{HPO}_{4^{-}} 0.5 \mathrm{~g}, \mathrm{MgSO}_{4}, 7 \mathrm{H}_{2} \mathrm{O}-0.2 \mathrm{~g}, \mathrm{NaCl}-0.1 \mathrm{~g}$, Yeast extract - $0.5 \mathrm{~g}$, Distilled water - 1 liter and Agar- $15 \mathrm{~g}$. The medium was prepared and was autoclaved at $121^{\circ} \mathrm{C}$ and 15 psi for 20 minutes. In the meantime, all accessories like Petridis and pipette $(1 \mathrm{ml})$ was also sterilized by autoclave. 


\subsection{Colony isolation}

The growth of Rhizobium was streaking on medium and incubated until pure growth was obtained. Finally, pure Rhizobium was cultured on slant medium as mother culture and stored in refrigerator. Then different biochemical test is to be done and new mother culture was done after 3-4 months. The colonies showing clear zones around them developed within 48 hours were transferred to agar slants of Yeast mannitol agar medium and allowed to grow at $30^{\circ} \pm 2^{\circ} \mathrm{C}$ for three days. The cultures were then repeatedly plated in the same agar medium till pure strains were obtained and finally 20 bacterial cultures were maintained in the Yeast mannitol agar medium.

\subsection{Estimation of bacterial population}

The viable cells were calculated by the following formula stated by Somasegaran and Hobben (Somasegaran and Hobben, 1985).

Number of cells $/ \mathrm{ml}(\mathrm{CFU} / \mathrm{ml})=[(($ Number of colonies $) \times($ Dilution factor $)\} \div($ Volume per drop $)]$

\subsection{Purification of isolates}

Six isolates of each Rhizobium were taken from respective cultured media and streaked on respective prepared plate's media. The streaked plates were incubated at $28^{\circ} \mathrm{C}$ for $2-4$ days. Repeated streaking was done until purification.

\subsection{Identification of Rhizobium isolates}

The isolates of Rhizobium obtained from soils were described according to their growth characteristics on solid and liquid Yeast Mannitol Agar media. Some morphological characters such as the shape, size, color, texture of colonies and ability to alter $\mathrm{pH}$ and some biochemical characters such as carbohydrate utilization and fermentation, gelatin and starch hydrolysis, Congo red dye absorption.

\subsection{Preparation and preservation of mother culture}

Purified isolates of Rhizobium were transferred into Yeast Mannitol Agar media and preserved for further study.

\section{Results and Discussion}

\subsection{Estimation of Rhizobia}

Bacterial populations of collected soils were determined and presented in Table 2. The results show the highest populations of Rhizobium $2.8 * 10^{6}$ were found in soil no. 1 (Aslampur, Charfashion, Bhola) and the lowest populations of Rhizobium $2.2 * 10^{6}$ were found in soil no. 2 (Jamla, Dumki, Patuakhali).

\section{Table 2. Bacterial population of Rhizobium from collected soil sample.}

\begin{tabular}{ll}
\hline Location & Rhizobium $\left(\mathbf{C F U g}^{-1}\right)$ \\
\hline 1. Aslampur, Charfashion, Bhola & $2.8^{*} 10^{6}$ \\
2. Ginnagor, Charfashion, Bhola & $2.6^{*} 10^{6}$ \\
3. Usmangonj, Charfashion, Bhola & $2.7 * 10^{6}$ \\
4. Srirampur, Dumki, Patuakhali & $2.3^{*} 10^{6}$ \\
5. PSTU Farm, Dumki, Patuakhali & $2.5^{*} 10^{6}$ \\
6. Jamla, Dumki, Patuakhali & $2.2 * 10^{6}$ \\
\hline
\end{tabular}

\subsection{Isolation of Rhizobium from saline soils of coastal region}

Six Rhizobium isolates were obtained from Non-saline soil of coastal region. They were designated as R1, R2, R3, R4, R5 and R6 respectively (Table 3).

Table 3. List of isolates from saline and non saline soil areas.

\begin{tabular}{lll}
\hline Soil No. & Isolate name & Location \\
\hline 1. & R1 & Aslampur, Charfashion, Bhola \\
2. & R2 & Ginnagor, Charfashion, Bhola \\
3. & R3 & Usmangonj, Charfashion, Bhola \\
4. & R4 & Srirampur, Dumki, Patuakhali \\
5. & R5 & PSTU Farm, Dumki, Patuakhali \\
6. & R6 & Jamla, Dumki, Patuakhali \\
\hline
\end{tabular}


3.3. Characterization of the isolates

Results of isolation as well as morphological and biochemical characteristics of isolates are presented below.

\subsubsection{Morphological characteristics}

Morphological characteristics of the isolates i.e. colony morphology have been presented in Table 4 and Table 5. The colony characteristics of isolates did not vary widely. All the isolates were found round shape, medium flat elevation, whitish colour, smooth surfaces, odour less, viscous consistency, opaque opacity with entire margin on Congo red yeast extract mannitol agar (CRYFMA) plates. All the isolates were found medium, small, large in size.

\subsubsection{Microscopic tests}

\subsubsection{Simple staining (shape of cells)}

The shape of the cells of rhizobia isolates are presented in Table 5. All the isolates were found short rod in shape. Vincent stated that Rhizobium was rod/ short rod shaped (Vincent et al., 1980).

\subsubsection{Motility test}

All the 6 isolates under study were found motile in nature. Vincent stated that Rhizobium was generally motile (Vincent et al., 1980).

\subsubsection{Gram reaction test}

All the 6 isolates have shown gram negative in reaction. Vincent stated that Rhizobium was gram negative (Vincent et al., 1980).

Table 4. Colony characteristics of Rhizobium isolates on Yeast Mannitol Agar media.

\begin{tabular}{lllllllll}
\hline \multicolumn{1}{c}{ Isolate } & Shape & Elevation & Odor & Margin & Surface & Opacity & Colour & Consistency \\
\hline R1 & Round & Medium flat & Odor less & Entire & Smooth & Opaque & Whitish & Viscous \\
R2 & Round & Medium flat & Odor less & Entire & Smooth & Opaque & Whitish & Viscous \\
R3 & Round & Medium flat & Odor less & Entire & Smooth & Opaque & Whitish & Viscous \\
R4 & Round & Medium flat & Odor less & Entire & Smooth & Opaque & Whitish & Viscous \\
R5 & Round & Medium flat & Odor less & Entire & Smooth & Opaque & Whitish & Viscous \\
R6 & Round & Medium flat & Odor less & Entire & Smooth & Opaque & Whitish & Viscous \\
\hline
\end{tabular}

Table 5. Morphological (microscopic) characteristics of Rhizobium isolates.

\begin{tabular}{llll}
\hline Isolate & Shape & Gram reaction & Motility \\
\hline R1 & Short rod & Gram negative & Motile \\
R2 & Short rod & Gram negative & Motile \\
R3 & Short rod & Gram negative & Motile \\
R4 & Short rod & Gram negative & Motile \\
R5 & Short rod & Gram negative & Motile \\
R6 & Short rod & Gram negative & Motile \\
\hline
\end{tabular}

\subsubsection{Biochemical tests}

Results of biochemical tests are presented below-

\subsubsection{Congo red absorption test}

From the Table 6 it was presented observed that all the bacterial isolates did not absorb Congo red at young stage but absorbed slightly when cultures became old. The isolates absorbed counter stain. Vincent et al. (1980) stated that Rhizobium was gram negative, rod shaped and generally motile. The isolates produce circular, low convex to convex, mucous and opaque white. The isolates were observed to lack the ability to absorb Congo red from yeast extract mannitol agar medium containing this dye. Similar result was observed by Barbar (Barbar $e l$ al., 1983). 
Table 6. Congo red absorption of different rhizobial isolates.

\begin{tabular}{llc}
\hline Isolate & & Congo red absorption \\
\cline { 2 - 3 } & Young culture & Old culture \\
\hline R1 & Not absorbed & Weakly absorbed \\
R2 & Not absorbed & Weakly absorbed \\
R3 & Not absorbed & Weakly absorbed \\
R4 & Not absorbed & Weakly absorbed \\
R5 & Not absorbed & Weakly absorbed \\
R6 & Not absorbed & Weakly absorbed \\
\hline
\end{tabular}

\subsubsection{BTB test}

All the bacterial isolates produced acid on BTB-YEMA plates. The results are presented in Table 7 . The growth of the all fast growers develops yellow color that results acidic in nature.

\subsubsection{Hofer's alkaline broth test}

Among the six isolates none had grown on Hofer's alkaline broth (Table 7).

Table 7. Biochemical observation of different rhizobial isolates.

\begin{tabular}{|c|c|c|c|c|}
\hline \multirow[t]{2}{*}{ Isolate } & \multicolumn{3}{|c|}{ BTB test } & \multirow{2}{*}{$\begin{array}{l}\text { Hoffer's alkaline test } \\
\text { Growth }\end{array}$} \\
\hline & Growth & Observation & Result & \\
\hline R1 & Fast growth & Yellow colour & Acidic & No growth \\
\hline $\mathrm{R} 2$ & Fast growth & Yellow colour & Acidic & No growth \\
\hline R3 & Fast growth & Yellow colour & Acidic & No growth \\
\hline $\mathrm{R} 4$ & Fast growth & Yellow colour & Acidic & No growth \\
\hline R5 & Fast growth & Yellow colour & Acidic & No growth \\
\hline R6 & Fast growth & Yellow colour & Acidic & No growth \\
\hline
\end{tabular}

\subsubsection{Growth on Different pH}

The growth responses of the Rhizobium isolates were investigated in the YEMA medium having 5 levels of $\mathrm{pH}$. The $\mathrm{pH}$ levels 4.0, 5.0, 6.0, 7.0 were created adding $\mathrm{HCl}$ solution and 8.0 adding $\mathrm{NaOH}$ as required. Results in the Table 8 show that all the isolates viz., R1, R2, R3, R4, R5 and R6 were heavy growers at pH 6.0 and 7.0. At pH 8.0 all isolates viz., R1, R2, R3, R4, R5 and R6 were found medium growth. But at $\mathrm{pH} 4$ and 5 isolates were found minimum to medium growth. At $\mathrm{pH} 5.0$ most of the isolates were found medium growth except R3. Kucuk found that rhizobia grew on pH levels 5 and 9 (Kucuk et al., 2006). Similarly Shraddha Bhatt also found that rhizobia were grown in YEM medium with pH values of 4, 5, 7 and 9 (Shraddha Bhatt et al., 2013).

Table 8. Effect of different pH on Rhizobium isolates in Yeast Mannitol Agar media.

\begin{tabular}{|c|c|c|c|c|c|}
\hline \multirow[t]{2}{*}{ Isolate } & \multicolumn{5}{|c|}{ Different pH } \\
\hline & 4 & 5 & 6 & 7 & 8 \\
\hline R1 & - & + & ++ & ++ & + \\
\hline $\mathrm{R} 2$ & + & + & ++ & ++ & + \\
\hline R3 & - & - & ++ & ++ & + \\
\hline $\mathrm{R} 4$ & - & + & ++ & ++ & + \\
\hline $\mathrm{R} 5$ & + & + & ++ & ++ & + \\
\hline R6 & + & + & ++ & ++ & + \\
\hline
\end{tabular}

$++=$ Heavy growth,$+=$ Medium growth, $-=$ Minimum growth

\subsubsection{Carbohydrate utilization}

Results of carbohydrate utilization by the isolates are presented in Table 9. The sign of carbohydrate utilization was observed from the growth and fermentation characteristics of the isolates in a given carbohydrate medium and the variation in growth was identified by measuring the optical density of the media. It was observed that the isolates R1 and R5 showed heavy growth in mannitol and sucrose. R2, R3, R4 and R6 showed minimum growth in mannitol and sucrose. It was also observed that all the isolates showed minimum growth in glucose and produced gas in carbohydrate media used. Chowdhury and Knan (1968) and Podder (1977) working with 
chickpea isolates also recorded similar results. Graham reported that most of the rhizobial strains utilized glucose, xylose and arabinose but lactose and sucrose were utilized by a few slow growing rhizobia (Graham et al., 1964).

Table 9. Carbohydrate utilization and fermentation by the strains.

\begin{tabular}{llll}
\hline Strain & Sucrose & Glucose & Mannitol \\
\hline R1 & ++ & + & ++ \\
R2 & + & + & + \\
R3 & + & + & + \\
R4 & + & + & + \\
R5 & ++ & + & ++ \\
R6 & + & + & + \\
\hline
\end{tabular}

$++=$ Heavy growth,$+=$ Minimum growth

\subsubsection{Gelatin hydrolysis}

Results in Table 10 show that R4, R5 and R6 isolates had the capacity to hydrolyse gelatin. On the other hand, R1, R2 and R3 gave negative result for gelatin hydrolysis. But Podder reported that none of the isolates from chickpea used in his study could hydrolyse gelatin (Podder et al., 1977).

\subsubsection{Strach hydrolysis}

R1, R2, R3, R4 and R6 of the isolates gave positive results for starch hydrolysis (Table 10). R5 of the isolates gave negative results for starch hydrolysis (Table 10). Halos developed around the bacterial colonies. Podder also noted that the isolates from chickpea failed to cause hydrolysis of starch (Podder et al., 1977).

\subsubsection{Catalase test}

Results in the Table 10 show that all of the test isolates gave positive results for catalase test. All of the isolates produced bubbles within a few seconds.

Table 10. Gelatin hydrolysis, starch hydrolysis and catalase test.

\begin{tabular}{llll}
\hline Isolates & Gelatin hydrolysis & Starch hydrolysis & Catalase test \\
\hline R1 & $(-)$ & $(+)$ & + \\
R2 & $(-)$ & $(+)$ & + \\
R3 & $(-)$ & $(+)$ & + \\
R4 & $(+)$ & $(-)$ & + \\
R5 & $(+)$ & $(+)$ & + \\
R6 & $(+)$ & + \\
\hline
\end{tabular}

$(+)=$ hydrolytic, $(-)=$ nonhydrolytic and $+=$ positive result

\subsubsection{Growth at different temperature conditions}

All the isolates showed good growth at temperature $28^{\circ} \mathrm{C}$ and $32^{\circ} \mathrm{C}$ (Table 11). Most of the isolates grew weakly (poor growth) at $14^{\circ} \mathrm{C}$ except R3, R5 and R6 isolates. At $22^{\circ} \mathrm{C}$ most isolates exhibited medium growth while three isolates (R3, R5 and R6) recorded poor growth. All the isolates grew at $38^{\circ} \mathrm{C}$. Only three isolates (R3, R5 and R6) showed medium growth at $38^{\circ} \mathrm{C}$ while rest three showed poor growth. At $45^{\circ} \mathrm{C}$ most isolates exhibited no growth while two isolates (R5 and R6) recorded very poor growth (Table 11).

Table 11. Growth of Rhizobium isolates in different temperature conditions.

\begin{tabular}{|c|c|c|c|c|c|c|}
\hline \multirow[t]{2}{*}{ Isolate } & \multicolumn{6}{|c|}{ Growth in different temperature condition } \\
\hline & $14^{0} \mathrm{C}$ & $22^{0} \mathrm{C}$ & $28^{0} \mathrm{C}$ & $32^{0} \mathrm{C}$ & $38^{0} \mathrm{C}$ & $45^{0} \mathrm{C}$ \\
\hline R1 & ++ & +++ & ++++ & ++++ & ++ & - \\
\hline $\mathrm{R} 2$ & ++ & +++ & ++++ & ++++ & ++ & - \\
\hline R3 & - & ++ & ++++ & ++++ & +++ & - \\
\hline $\mathrm{R} 4$ & ++ & +++ & ++++ & ++++ & ++ & - \\
\hline R5 & - & ++ & ++++ & ++++ & +++ & + \\
\hline R6 & - & ++ & +++ & ++++ & +++ & + \\
\hline
\end{tabular}

- = No growth,$+=$ Very poor growth,$++=$ Poor growth,$+++=$ Medium growth,$++++=$ Good growth 


\section{Conclusions}

It is concluded from the present study that the utilization of as bioinoculants may increase the fix atmospheric nitrogen in soil. It avails to minimize the nitrogen fertilizer application, minimize environmental pollution and promotes sustainable agriculture. The study ventilated that Rhizobium isolated from rice rhizosphere could be utilized for sustainable rice crop production system in Bangladesh. Their performance under green house and field conditions should be assessed afore being recommended for biofertilizer production and commercial applications.

\section{Conflict of interest}

None to declare.

\section{References}

Barbara EK and AL Thomas, 1983. Congo red absorption by Rhizobium leguminosarum. Appl. Environ. Microbiol., 45: 340-342.

Chowdhury MS and AKM Khan, 1968. Cultural characteristics of certain native strains of root nodule bacteria of East Pakistan. J. Soil Sci., 4: 21-29.

Franche C, K Lindstrom and C Elmerich, 2009. Nitrogen fixing bacteria associated with leguminous and nonleguminous plants. Plan Soil, 321: 35-59.

Graham PH, 1964. Diagnostic features in the characterisation of the root nodule bacteria of legumes. Plant and Soil, 20: 383-396.

Kiers ET, RA Rousseau, SA West and RF Denison, 2003. Host sanctions and the legume-rhizobium mutualism. Nature, 425: 79-81.

Kucuk C, M Kivanc and E Kinac, 2006. Characterization of Rhizobium sp. Isolated from Bean. Turk. J. Biol., 30: 127-132.

Podder AK and AKM Habibullah, 1982. Performance of some isolated native rhizobial strains on the production and nodulation of chickpea. Bangladesh J. Soil Sci., 18: 30-35.

Shraddha Bhatt DrRV, H Vyas, N Shelat and J Sneha, 2013. Isolation and Identification of Root Nodule Bacteria of Mung Bean (Vigna radiate L.) for Biofertilizer Production. International Journal of Research in Pure and Applied Microbiology, 3: 127-133.

Somasegaran P and HJ Hobben, 1985. Methods in legume-Rhizobium technology. NifTAL project and MIRCEN, Department of Agronomy and Soil science, University of Hawaii.

Vincent JM, PS Nutman and FA Skinner, 1980. Some General Techniques and Procedures - Identification and Classification of Rhizobium. Research for development seminar on "Nitrogen Fixation by Legumes for Tropical Agriculture" held in Canberra. Australia, during Nov.-Dec. 1980.

Vitousek PM, 1997. Human alteration of the global nitrogen cycle: sources and consequences. Ecol. Appl., 7 : 737-750. 